Société d'histoire de la révolution de 1848 et des

révolutions du XIXe siècle

45 | 2012

Le quotidien des techniques

\title{
Laurence GUIGNARD, Pascal RAGGI et Étienne THÉVENIN [dir.], Corps et machines à l'âge industriel
}

Rennes, Presses universitaires de Rennes, 2011, 324 p. ISBN :

978-2-7535-1395-2. 18 euros.

\section{Manuel Charpy}

\section{(2) OpenEdition}

Édition électronique

URL : http://journals.openedition.org/rh19/4359

DOI : $10.4000 /$ rh 19.4359

ISSN : $1777-5329$

Éditeur

La Société de 1848

Édition imprimée

Date de publication : 31 décembre 2012

Pagination : 191-193

ISSN : 1265-1354

\section{Référence électronique}

Manuel Charpy, «Laurence GUIGNARD, Pascal RAGGI et Étienne THÉVENIN [dir.], Corps et machines à l'âge industriel », Revue d'histoire du XIXe siècle [En ligne], 45 | 2012, mis en ligne le 02 avril 2013, consulté le 22 septembre 2020. URL : http://journals.openedition.org/rh19/4359 ; DOI : https:// doi.org/10.4000/rh19.4359

Ce document a été généré automatiquement le 22 septembre 2020

Tous droits réservés 


\section{Laurence GUIGNARD, Pascal RAGGI et Étienne THÉVENIN [dir.], Corps et machines à l'âge industriel}

Rennes, Presses universitaires de Rennes, 2011, 324 p. ISBN :

978-2-7535-1395-2. 18 euros.

Manuel Charpy

\section{RÉFÉRENCE}

Laurence GUIGNARD, Pascal RAGGI et Étienne THÉVENIN [dir.], Corps et machines à l'âge industriel, Rennes, Presses universitaires de Rennes, 2011, 324 p. ISBN :

978-2-7535-1395-2. 18 euros.

1 Les corps au travail, meurtris par les machines, ont fait ces dernières années l'objet d'une attention renouvelée ${ }^{1}$. Mais les travaux qui tentent d'embrasser toute la diversité des relations entre corps et machines demeurent rares. Si dans cet ouvrage le monde $\mathrm{du}$ travail tient une grande place, il considère aussi les machines médicales, de transport, de loisir, etc., questionnant la manière dont les machines délassent, soutiennent, prolongent ou fatiguent les corps ; mais aussi, en retour, comment les corps sont modelés par les machines et en deviennent parfois le prolongement. On l'aura compris, il s'agit d'une histoire des gestes et des postures attachés à la machine à l'âge industriel - une histoire de l'ergonomie au sens large -, et notamment au XIX ${ }^{\mathrm{e}}$ siècle, moment où la machine change le quotidien des corps et où l'on s'attache, avant la taylorisation, à analyser et fixer les gestes professionnels. On songe à l'ouvrage de Denis Poulot ${ }^{2}$ qui détermine par l'image et le texte les gestes du mécanicien mais aussi aux études qui après 1880 tentent de décrypter les gestes professionnels ${ }^{3}$. Conceptions mécanistes et néo-hippocratiques du corps et technologie nouent tout au long du siècle un dialogue nouveau et complexe. Les cinq parties du livre (machines de santé; machines de mort; machines de travail ; machines de loisir ; régénération et fatigues 
des corps) permettent de dépasser une approche qui réduirait les relations corpsmachine au seul asservissement. Les articles, les plus nombreux, qui concernent le XIX ${ }^{\mathrm{e}}$ siècle, sont aussi les plus originaux - sans doute parce que le travail à la chaîne focalise, pour le $\mathrm{XX}^{\mathrm{e}}$ siècle, les regards.

2 Si quelques articles apparaissent à la marge, le travail de problématisation permet la multiplication des points de vue et des terrains tout en gardant une grande cohérence. Fabien Knittel montre ainsi comment dans la première moitié du XIX ${ }^{\mathrm{e}}$ siècle l'introduction de la charrue sans avant-train de l'agronome Dombasle oblige à réformer postures et gestes paysans. C'est à l'aide du dynamomètre qu'il démontre les avantages de sa charrue contre les habitudes dont les gestes sont sans aucun doute l'incorporation la plus durable. Pour le monde de l'industrie, Thomas Le Roux s'interroge sur la manière dont les médecins-hygiénistes observent et préservent le corps ouvriers soumis aux machines. Si elles sont parfois le moyen de protéger les corps des substances toxiques et des taches épuisantes, les enquêtes sur les lésions qu'elles causent n'arrivent que dans les années 1860 , bien après les enquêtes sur les conditions de vie des ouvriers, et les solutions - masques, vêtements professionnels (qui mériteraient une enquête)... - sont parcimonieusement préconisées. Ce sont les ingénieurs sur le terrain qui proposent des solutions, ce qui invite à penser que les médecins-hygiénistes arment le bras de l'industrie bien plus qu'ils ne protègent les corps en proie aux machines. Laurent Heyberger s'intéresse à la stature des corps au XIX ${ }^{e}$ siècle, en adoptant une perspective d'histoire anthropométrique qui a le mérite de battre en brèche les idées reçues sur les effets corporels de l'industrialisation mais peine à dire quelque chose des relations corps-machines puisque dans le textile observé, le travail à domicile rencontre aussi la mécanisation par la réduction des machines et la miniaturisation des moteurs. Enfin, Nicolas Hatzfeld revient sur la naissance de la chaîne, fruit de la coordination des machines et du chronométrage.

3 L'ouvrage ne se cantonne toutefois pas au monde du travail. Nicole Edelman montre qu'au-delà du charlatanisme du baquet Mesmer et des machines électriques qui lui succèdent, ces machines conduisent à l'instauration d'une distance entre médecin et patient dans laquelle nait une nouvelle forme de diagnostic. Dans la même logique, Alexandre Klein analyse les machines à enregistrer les soubresauts du psychisme qui sont aussi des machines à provoquer l'hypnose. À côté du monde médical, se développe un marché prospère des machines à guérir. Denise Bernuzzi de Sant'Anna observe ainsi la manière dont le Veedee, petite machine à masser nécessairement miraculeuse, s'insère dans une société brésilienne partagée entre désir de modernité et soins traditionnels.

4 L'autre champ exploré par cet ouvrage est celui des rapports entre machine et morts : machines de mort pour la Grande Guerre mais aussi traitement des cadavres. Les abattoirs ont été un des hauts lieux de la mécanisation du travail ; rien de surprenant à ce que la question, comme le montre Anne Carol, se pose pour le traitement des cadavres humains. Mais alors qu'hygiénistes et édiles rêvent de cadavres rendus invisibles et inoffensifs par leur transport en train et leur crémation, la population rejette le crématorium du Père-Lachaise, le premier édifié en 1889. Le rapport au corps peut se faire radical avec les machines infernales dont les explosions ponctuent le XIX ${ }^{\mathrm{e}}$ siècle. Karine Salomé montre bien l'évolution de la sensibilité et des usages politiques des corps victimes : les corps mutilés par la machine de Fieschi sont exposés en place publique alors qu'en 1858 les corps des victimes d'Orsini sont à peine exhibés. De 
l'embaumement à l'exposition dans le Petit Journal des attentats anarchistes à la dynamite, du martyr à l'innocente victime, si le statut de la victime change, les machines conservent leur capacité à terrifier. D'autres machines létales mériteraient d'être considérées, de la guillotine au pistolet de ville.

5 La machine est aussi un pourvoyeur de nouvelles expériences qui impliquent corps et machines, comme celle de la vitesse, connue depuis les travaux de Christophe Studeny ${ }^{4}$. Philippe Gaboriau analyse le vélocipède puis la bicyclette comme une expérience physique nouvelle de l'espace et Jérémie Legroux montre comment les débuts de l'aviation bouleversent les sensations au point d'inquiéter les putatifs passagers.

Les machines modèlent aussi les corps. Si l'ouvrage néglige par exemple les machines de musculation - qu'on songe au succès des bicyclettes d'appartement après 1890 -, il s'arrête, même si la photographie est absente, sur la manière dont les machines figurent les corps et les médiatisent. C'est le cas des physionotraces analysés par Guillaume Mazeau: la mécanique qui produit ces profils est censée conférer aux portraits une ressemblance nouvelle. Plus encore, elle permet les multiples qui participent à la fabrication des identités sociales et publicisent les corps dans le nouvel espace public démocratique de la fin du XVIII ${ }^{\mathrm{e}}$ siècle et du début du XIX ${ }^{\mathrm{e}}$ siècle. À l'autre extrémité du siècle, le cinéma burlesque, analysé par Rae Beth Gordon, semble à la croisée de la gestuelle des "dégénérés » observée par le monde médical et des pantomimes et trucages d'un art mécanique, le cinéma.

7 Si quelques articles apparaissent trop succincts et si l'on est tenté de pointer les relations corps-machines absentes de ce panorama - machines sexuelles, machines médicales domestiques, prothèses, jouets, etc. - c'est avant tout parce que cet ouvrage dessine un nouveau territoire à explorer.

\section{NOTES}

1. On songe notamment à : Caroline Moriceau, Les douleurs de l'industrie. L'hygiénisme industriel en France, 1860-1914, Paris, Éditions de l'EHESS, 2010 ; François Jarrige, Au temps des "tueuses de bras » ..., op. cit.; Thierry Pillon, Le corps à l'ouvrage, Paris, Stock, 2012 et Michelle Perrot, Mélancolie ouvrière, Paris, Grasset, 2012.

2. Denis Poulot, Méthode d'enseignement manuel pour former un apprenti mécanicien, Paris, Monrocq, 1889.

3. Charles Hacks, Le geste, Paris, Flammarion, 1892.

4. Christophe Studeny, L'invention de la vitesse, France XVIII ${ }^{e}-X X^{e}$ siècles, Paris, Gallimard, 1995. 